\title{
Der geistig gestörte Künstler im Werk E. T. A. Hoffmanns vor dem Hintergrund zeitgenössischer psychologischer Theorien
}

von Vickie Ziegler

\section{Zusammenfassung}

E.T. A. Hoffmann, einer der grössten Schriftsteller der deutschen Romantik, hat sehr viel zeitgenössisches psychologisch-psychiatrisches Wissen und Denken in sein dichterisches Werk eingearbeitet. Eine tiefsitzende Hypochondrie verstärkte seine Neigung zum Studium medizinischer Werke. In unserem Zusammenhang sind vor allem die Autoren Pinel, Reil, Kluge und G. H. Schubert wichtig. An der Figur des Goldschmiedes Cardillac aus dem "Fräulein von Scuderi», der seine Kunden ermordet, wird dargelegt, wie Hoffmann wissenschaftliche Konzepte dichterisch gestaltet; bei dem ausgewählten Beispiel geht es vor allem um den Mesmerismus und die Nachwirkung vorgeburtlicher Einflüsse.

Der folgende Aufsatz soll zeigen, wie die Berücksichtigung von Hoffmanns medizinisch-psychologischer Lektüre dem Literaturkritiker ein besseres Verständnis seiner Werke erschliessen kann.

Während der romantischen Periode in der deutschen Literatur (17901830) beschäftigten sich viele Schriftsteller mit dem Begriff der Geisteskrankheit und der richtigen Einstellung dem gefährdeten Menschen gegenüber sowie mit den ethischen Problemen, die aufgrund der neuen Entdeckungen auf dem Gebiet der Medizin zu Tage traten. Bei Ernst Theodor Amadeus Hoffmann (1776-1822) sieht man vielleicht am deutlichsten, wie diese Tendenzen zusammenlaufen. Selbst recht bewandert in der neuesten Literatur über Geisteskrankheit, interessierte sich Hoffmann sehr für die verschiedenen Arten des Wahnsinns. Diese Anteilnahme führte ihn dazu, nicht nur die neueste Medizinwissenschaft, sondern auch Diskussionen der medizinischen Ethik in sein künstlerisches Werk einzuarbeiten. In einem seiner Hauptwerke, Die Serapions-Brüder, schafft die Rahmen-/Binnengeschichtsstruktur ein besonders gut geeignetes Gerüst dafür. Die Rahmenfiguren, eine fiktive Gruppe Intellektueller, meist Künstler, treffen sich, um sich jeweils die neueste Novelle eines Mitglieds vorlesen zu lassen. In den 
Rahmen dieses Werkes stellt Hoffmann immer wieder Krankheitsfälle von wahnsinnigen Menschen, von denen er oft durch Bekannte, die Ärzte waren, erfuhr oder über die er ausführlich gelesen hat. Die Binnengeschichten, die solche Stoffe behandeln, dienen verschiedenen Zwecken, wie z. B. der Bestätigung des medizinischen Stoffes im Rahmen oder in der Darstellung einer Situation, in der sich medizinisch-ethische Probleme zu einer Sache auf Leben und Tod entwickeln.

Anhand dieses Werkes will dieser Aufsatz Hoffmanns Verständnis des Wahnsinns und der zeitgenössischen medizinischen Schriften besprechen, vor allem auf dem Gebiet des Magnetismus und der vorgeburtlichen Einflüsse. Diese Themen führen bei Hoffmann, dem Juristen, zu Fragen der Zurechnungsfähigkeit, die auch in einigen Novellen erörtert werden. Obgleich Hoffmanns medizinische Interessen im allgemeinen viel kritische Beachtung auf sich gezogen haben, ist die Bedeutung dieses Fachwissens für die Interpretation einzelner Werke noch bei weitem nicht ermessen ${ }^{1}$. Am Beispiel des schwierigen Hauptcharakters Cardillac in Hoffmanns «Fräulein von Scuderi» in den Serapions-Brüdern wird gezeigt, dass durch das Studium der betreffenden Fachwerke rätselhafte Züge in Cardillacs Charakter erleuchtet und neue Einsichten für die Kritik gewonnen werden können.

Ein ständiger Hypochonder, war Hoffmann seinem ärztlichen Bekanntenkreis wohl eine anhaltende Plage. Nicht nur immerwährende Verdauungsschwierigkeiten, die durch eine beinah ausschliessliche Diät von Wein, Punsch und Branntwein weiter gereizt wurden, sondern auch eine ererbte Tendenz zum Wahnsinn trieb ihn dazu, in medizinischen Büchern eifrig zu lesen ${ }^{2}$. Aus seiner persönlichen Anteilnahme heraus wurde Hoffmann zu einem der ersten deutschen Schriftsteller, die das Thema des ererbten Wahnsinns in der schönen Literatur besprechen ${ }^{3}$.

Hoffmanns nagende Angst um seine prekäre Geistesgesundheit nährte sich an dem verbreiteten Fachinteresse an diesem Thema. Die neue Einschätzung des Wahnsinns entstand zuerst bei dem schottischen Mediziner John Brown (1735-88); bald übten seine Ideen einen grossen Einfluss auf die deutschen Romantiker und deutschen Ärzte aus, zu denen sich Hoffmanns Freunde in Bamberg, wie Dr. Adalbert Marcus, zählten ${ }^{4}$. Brown glaubte, dass es zwei krankhafte Zustände gäbe: den sthenischen Zustand der Überreiztheit und den asthenischen der Nervenschwäche. Von diesem Konzept ausgehend, erkannten andere bald Analogien zwischen Körper und Seele, zwischen der inneren und der äusseren Welt ${ }^{5}$. Solche Vergleiche lieferten die Basis für eine positive Bewertung des Wahnsinns, weil der asthenische 
Mensch schnell ein höchst positives Symbol der Romantik wurde, ein Träger der höheren geistigen Werte. Die Nacht wurde auch als asthenisch betrachtet, so wie die Unvorsichtigkeit, die zu dieser Zeit den Menschen oft heimsucht; der Wahnsinn galt als die höhere Form dieses Leichtsinns ${ }^{6}$. Von nervösen Krankheiten, durch die die Gefühle leicht erregt wurden, glaubte man, dass sie die Kranken geistig verfeinern; der Wahnsinn wurde als <indirekte Asthenies angesehen ${ }^{7}$. Viele Ärzte widmeten sich dem Unterbewusstsein und dessen Erscheinungen, unter anderen Gotthilf H. Schubert (1780-1860), Johann Christian Reil (1759-1813) und Carl Gustav Carus (1789-1869 $)^{8}$. Ihre Interessen lassen sich mit denen vieler romantischer Schriftsteller vergleichen, Hoffmann eingeschlossen. Schubert, in seinen Ansichten von der Nachtseite der Naturwissenschaft, deutete an, dass der kranke Mensch imstande sei, dem eigenen Geist näherzukommen ${ }^{9}$. Diese Einschätzung des Wahnsinns tauchte in den Schriften Hoffmanns (unter anderen) auf und hatte als Folge, dass der Wahnsinn in der Literatur oft sowohl mit positiven als auch negativen Aspekten ausgestattet wurde ${ }^{10}$. Dieses romantische Konzept, dass der Mensch in einem Zustand der Irrationalität fähiger sei, zu wesentlichen Wahrheiten über sich selbst zu gelangen, taucht sogar bei Freud auf, der behauptete, dass die Depression ziemlich genaue Selbstkritik herbeiführen kann, Einblicke, die nur dem Kranken gegönnt sind ${ }^{11}$.

Philippe Pinel (1745-1826) und J. C. Reil übten einen grossen Einfluss auf Hoffmanns Begriff der Geisteskrankheit aus. Beide Wissenschaftler wiesen die mechanistische Behandlung des Wahnsinns zurück, die im frühen achtzehnten Jahrhundert beliebt war. Pinel betrachtete Geisteskrankheit als Resultat der Vererbung und der Gemütserlebnisse; infolgedessen konnten nur Ärzte, die auch für das menschliche Herz Verständnis aufbringen konnten, diese Krankheit richtig begreifen. Reil bot eine systematische Stellungnahme zur Behandlung der Geisteskrankheit in seine Rhapsodien über die Anwendung der psychischen Curmethode auf Geisteszerrüttungen (1803). Wenn positive psychologische Einflüsse Geisteskrankheiten heilen können, dann könnten negative Einflüsse sie verursachen, eine Situation, die in Hoffmanns Schriften immer wieder auftaucht, z. B. bei seinem Cardillac.

Der Glaube an die Kraft des Magnetismus, das Wahrnehmungsvermögen und Bewusstsein zu erweitern, sowie die Behauptung, dass er die Existenz versteckter Geisteskräfte aufdecken könnte, übten einen grossen Reiz auf 
die Romantiker aus, weil man damals allgemein glaubte, dass das Unterbewusstsein die Basis sei, auf der eine Kenntnis des ganzen Menschen beruhe ${ }^{12}$. Der Magnetismus und sein Potential auf der Seite des Guten wie des Bösen waren beliebte Unterhaltungsthemen in dem Kreis der Intellektuellen, die Hoffmann kannte. Die intensive Forschung der deutschen Ärzte hielt der Öffentlichkeit dieses Konzept ständig vor Augen, zumal Informationen über die neuen psychischen Wunderkuren immer vorhanden waren, nicht nur in Fachzeitschriften, sondern auch in den Zeitungen ${ }^{13}$.

Die übertriebene Macht, die man irrigerweise dem Magnetiseur zuschrieb, war zugleich eine Quelle der Begeisterung und der Beunruhigung ${ }^{14}$. Schon im späten 18. Jahrhundert war es klar, dass der magnetisierte Mensch der Beeinflussung in einem Zustand der gesteigerten Aufnahmefähigkeit und Empfänglichkeit gegenüber stand ${ }^{15}$. Dazu glaubte man im frühen 19. Jahrhundert auch noch, dass der Magnetiseur das Subjekt allein durch seine Gedanken beherrschen könnte ${ }^{16}$.

Der Glaube, dass der Kranke in einem exaltierten Bewusstseinszustand sei, kommt oft in der Fachliteratur der damaligen Zeit vor ${ }^{17}$. Im folgenden Auszug aus den Serapions-Brüdern wird diese Lehre relativiert:

«Es ist gewiss, dass es erhöhte Zustände gibt, in denen der Geist, den Körper beherrschend, mächtig wirkt. [...] Wir erschauen das mit aller Kraft unseres vollen Fassungsvermögens, was tief in unserer Seele regungslos schlummerte; [...] Aber gewiss ist es auch, dass solch ein Zustand irgendeine Abnormität in dem Verhältnis des psychischen und physischen Prinzips voraussetzt. [...] Der Geist nutzt die Ohnmacht seines Mitherrschers und macht ihn [...] zum dienenden Vasallen. (S.263/4) $)^{18} .{ }^{\prime}$

Ein erweitertes Bewusstsein setzt also immer voraus, dass es eine Abnormität im Verhältnis zwischen Seele und Körper gibt. Diese Interpretation führte für Hoffmann in viele Richtungen, weil sie künstlerisches Schaffen mit Krankheit verband, so wie in dem Fall Cardillacs. Alle exaltierten Zustände bei seinen Charakteren deuten auf Unausgeglichenheit, einen gefährlichen Zustand, der dem gesunden Menschen nichts ausmacht, den Zerrissenen aber gefährdet ${ }^{19}$. Deswegen ist der exaltierte Mensch immer in einer prekären Lage: Einerseits ist er fähig, die tiefsten Geheimnisse zu begreifen, andererseits sind die Klippen des Wahnsinns oder der totalen Zerstörung nicht weit weg ${ }^{20}$. Weil der exaltierte Mensch den verborgenen Mächten der Seele gegenüber offen war, war der Magnetiseur in der Lage, seinen eigenen Vorteil zu suchen, ohne Rücksicht auf das Wohl des Schwachen; auch aus diesem Grund hatte Hoffmann Angst vor der Macht des Magnetiseurs, sei er Mensch oder Objekt ${ }^{21}$. 
Dieser Hintergrund erleichtert das Verständnis eines der schwierigsten Charaktere in Hoffmanns Schaffen: des verbrecherischen, wahnsinnigen Juweliers Cardillac im prärevolutionären Frankreich. René Cardillac ist die Hauptfigur einer Binnengeschichte in den Serapions-Brüdern, «Das Fräulein von Scuderi.» Cardillac war der berühmteste Juwelier seiner Zeit; seine Erzeugnisse waren bei der obersten Schicht des Adels begehrt. Aber so erhaben und bekannt die Kunden auch sein mochten, Cardillac fing an, sich zu überzeugen, dass nur er selber würdig war, seine Juwelen zu besitzen. Die Philister, die dem Künstler die gebührende Würdigung versagen, stören jeden Begabten, aber dieses Gefühl nimmt bei Cardillac ein ungeheures Ausmass an. Nicht nur hält er sich für den einzigen, der seine Goldarbeit richtig zu schätzen weiss, sondern die Kunden müssen sterben, weil sie nicht dazu imstande sind ${ }^{22}$. Cardillac erzählt, wie der Gedanke, dass ein Mitglied des Hofes ein Stück von ihm einer Operntänzerin schenken wollte, ihn zu seinem ersten Mord trieb (S.693-4). Er rechtfertigt seinen Diebstahl und Mord mit der Ausrede, dass diese Schätze sonst an unwürdige Empfänger vergeudet würden (663).

Verbunden mit dieser Verzerrung des künstlerischen Bewusstseins ist sein Gelüst nach Edelsteinen und -metallen, das bei ihm zur Manie wird. Diese idée fixe, die durch vorgeburtliche Einflüsse verursacht worden ist, zerstört ihn. Cardillacs unersättlicher Hunger nach Juwelen ist der Grund, warum er Goldschmied wurde, nicht etwa die Liebe zur Kunst (692). Sein ausserordentliches Können tarnt die grundlegende Perversion, die sein «böser Stern», wie er es nennt, verursacht. In seiner autobiographischen Darstellung verrät Cardillac die Quelle seiner Besessenheit mit Edelsteinen und Edelmetall, ein Erlebnis, das seine schwangere Mutter vor seiner Geburt mit einem Ritter hatte, der eine Goldkette mit Diamanten trug (692). Cardillac erinnert sich, dass ihn seit der frühesten Kindheit Diamanten und Gold am heftigsten anzogen; schon damals stahl er diese Dinge, wenn er ihrer sonst nicht habhaft werden konnte (692). Cardillac wird, wie Elis Fröbom in «Die Bergwerke zu Falun», (auch in den Serapions-Brüdern), von Edelsteinen und -metallen bezaubert.

Obgleich Kritiker Ähnlichkeiten zwischen den beiden Charakteren bemerkt haben, ist niemand der tieferen Verbindung mit den Schriften G. H. Schuberts nachgegangen ${ }^{23}$. Nach Schubert hat Gold einen gewaltigen Einfluss auf den Menschen in einem magnetischen Zustand, was möglicherweise seine Gier danach erklärt ${ }^{24}$. Kluge bemerkte, dass Experimente mit grossen Mengen von Metallen und deren Wirkung auf eine im magnetischen 
Zustand befindliche Person zuerst Wärme, danach Angst und schliesslich Zuckungen hervorriefen ${ }^{25}$. Eine solche Anziehungskraft brachte Fröbom seinem Untergang entgegen, genauso wie Cardillac.

Cardillacs ergreifende Beschreibung, wie er so weit kam, dass er die Kunden umbrachte, zeigt den Grad seiner Besessenheit. Um früheren Schwierigkeiten aus dem Wege zu gehen, hatte er gelernt, wie er den Trieb zum Juwelendiebstahl unterdrücken konnte. Aber der alte, unwiderstehliche Drang kam zurück. Sobald er mit einem Auftrag fertig war und die Arbeit ablieferte, packten ihn Schlaflosigkeit und Depression, die ihn seiner Gesundheit beraubten. Die Gestalt des Kunden tauchte vor seinem geistigen Auge auf, und eine Stimme flüsterte ihm zu, dass die Juwelen ihm gehörtenwozu brauchte ein Toter Diamanten? Da Cardillac als überaus aufrichtiger Mensch bekannt war, konnte er in herrschaftlichen Häusern ein- und ausgehen. So war es ihm ein leichtes, seine eigenen Erzeugnisse zu stehlen.

Der Diebstahl allein hat aber die inneren Stimmen nicht zum Schweigen bringen können. Zu dieser Zeit kaufte er sich einen Teil eines Hauses in Paris, das einen geheimen Eingang hatte. Dieser Bau ermöglichte es ihm, den inneren Dämonen zu folgen, denn der Mord wurde dadurch wesentlich erleichtert. Der erste Mord brachte ihm eine solche seelische Ruhe, dass ihm jetzt erst recht klar wurde, was sein böser Stern von ihm wollte. Entweder er gab nach, oder er musste zugrunde gehen; deswegen beging er einen Serienmord, der ganz Paris in Schrecken versetzte. Als Folge dieser Besessenheit weigerte er sich, einige Aufträge anzunehmen, weil er den Auftraggeber mochte und ihn nicht umbringen wollte (692-94).

Cardillacs Verbrechen führt zu einem anderen Thema, das für Hoffmann mit dem Magnetismus eng verbunden war, nämlich die Zurechnungsfähigkeit. Wie die vorangehende Besprechung andeutete, glaubten Hoffmann und viele seiner Zeitgenossen, dass der magnetisierte Mensch keine Verantwortlichkeit für sein Tun hatte. Wie andere Charaktere Hoffmanns, die sich im magnetischen Zustand befanden, wird Cardillac von inneren Stimmen heimgesucht. Wenn er von Edelmetallen und -steinen magnetisiert wird, vermindert dieser Umstand seine persönliche Verantwortlichkeit; man muss aber fragen, wie dieser Magnetismus zustande kam. Die Antworten liegen in dem anderen medizinischen Zustand, den Hoffmann in dieser Novelle behandelt.

Der Magnetismus mit seiner Herrschaft über den Geist des Subjekts und die dazugehörige Verminderung der persönlichen Verantwortlichkeit haben Beziehungen zu einem anderen wichtigen Thema in Cardillacs Leben: der Rolle 
des vorgeburtlichen Einflusses ${ }^{26}$. Während ihrer Schwangerschaft sah Cardillacs Mutter auf einem Hoffest einen Ritter, der mit einer glänzenden, juwelenbesetzten Kette geschmückt war. Jener Herr, der ihr vor der Heirat ohne Erfolg nachgelaufen war, kam ihr jetzt wie ein überirdisches Geschöpf vor. Der Kavalier, der jetzt auf mehr Glück hoffte, näherte sich ihr, umarmte sie und fiel, als sie nach der Kette griff, tot zu Boden. Das Gold und die Juwelen haben die Frau eindeutig magnetisiert, denn sie scheint wie erstarrt beim Anblick des Ritters und seiner Kette ${ }^{27}$. Aber ihr potentieller Liebhaber, wie der magnetisierte und magnetisierende Graf in «Der unheimliche Gast», stirbt, ehe er in den Genuss des Vorteils ihres magnetisierten Zustands kommt. Der Leichnam des Ritters trägt die charakteristischen Züge der Leichen von anderen bekannten Magnetiseuren in Hoffmanns Novellen ${ }^{28}$.

Das erschreckende Erlebnis hat die schwangere Frau schwer mitgenommen. Cardillac behauptete, dass das Grausen ihn im Mutterleib heimgesucht hätte, und beschreibt die Folgen als seinen «bösen Stern» (692). Cardillacs Deutung entspricht einem unter Laien und einigen Ärzten weitverbreiteten Glauben an vorgeburtliche Einflüsse. Obgleich die Existenz solcher Einflüsse im späten 18. Jahrhundert in medizinischen Kreisen höchst umstritten war, glaubte Kluge, den Hoffmann sehr bewundert, daran. Er meinte, dass vorgeburtliche Einflüsse besonders wahrscheinlich waren, wenn während der Schwangerschaft Magnetismus vorhanden war. Dazu erschien auch noch 1809, also kurz vor der Entstehung dieser Novelle, eine leidenschaftliche Verteidigung der vorgeburtlichen Einflüsse von einem gewissen D.H.G. Wüstnei. Dieser stellte magnetische und elektrische Beziehungen zwischen der Mutter und dem Kind in ihrem Leibe fest. Ein solcher Glaube scheint auch hier mitzuspielen: der magnetische Blick wird auf das Gold und die Juwelen übertragen, die dann Cardillac zeit seines Lebens beherrschen ${ }^{29}$.

Einer der bedeutenden Aspekte des Magnetismus und der vorgeburtlichen Einflüsse ist die Rolle des Schicksals, des Menschen als Opfer. Cardillac weist darauf hin, dass er mit den inneren Stimmen gerungen habe und dass seine mörderischen Instinkte bei weitem nicht seine ganze Persönlichkeit ausmachten (696). Die von aussen auf ihn einwirkenden Kräfte infizierten seinen geistigen Zustand und riefen dabei eine idée fixe hervor. Cardillac beschreibt eine innere Stimme, die ihm rät, die Juwelen zurückzunehmen. Die Tatsache, dass innere Stimmen ihm etwas zuflüstern, könnte ein weiterer Beweis dafür sein, dass Cardillac unter einem magnetischen Einfluss steht, denn auch Elis Fröbom, der von den Edelsteinen magnetisiert und so ins Verderben gestürzt wurde, hörte Stimmen. Weitere Indizien dafür, dass 
Cardillac seine mörderischen Gelüste nicht für sein wahres Ich hält, finden sich in seinem Geständnis, in dem er das Gefühl ausdrückt, dass seine unsterbliche Seele keinen Anteil an den Verbrechen habe (696).

Diese Analyse zeigt, dass man Cardillacs schwierigen Charakter leichter enträtseln kann, wenn man den medizinhistorischen Hintergrund berücksichtigt. Vom kritischen Standpunkt aus gesehen, verführt ein solcher Charakter dazu, Cardillac als tragische Künstlerfigur etwas zu verherrlichen oder aber von Begriffen wie Erbschuld zu reden ${ }^{30}$. Unsere Ausführungen deuten jedoch darauf hin, dass Hoffmanns Darstellung des verrückten Juweliers seine Quelle in der Problematik des medizinischen Wissens der damaligen Zeit hatte und von dort aus verstanden werden muss.

Da Hoffmann Cardillacs Bild mit vorgeburtlichen und magnetischen Einflüssen malt, verfolgt er wohl auch den Zweck, das Ausmass der von unsichtbaren Kräften verursachten Besessenheit darzustellen, wie er es bei anderen unglücklichen Charakteren in diesem Werk tat. Selbst bei Cardillac, dem Schlimmsten in der Schar der Besessenen, spürt man von der Seite Hoffmanns ein angeborenes Mitfühlen, das wahrscheinlich aus der Furcht entstand, dass er selbst in denselben Abgrund stürzen könnte. Seine Einfühlsamkeit hatte als weitere Quelle den Glauben, dass alle Künstler Gefahr liefen, geisteskrank zu werden, um schaffen zu können. Angst hatte er auch vor den geheimen Mächten, die den Geist beherrschen könnten, Mächten, die gewissenlose Menschen ausnutzten. Den Schriften dieses laienhaften Medizin-Enthusiasten ist nicht nur viel fachmännisches Wissen der damaligen Zeit einverleibt, sondern sie weisen auch auf ethische Fragen der Pschiatrie voraus. Themen wie der Zusammenhang zwischen Behandlung und schöpferischer Fähigkeit und das Verhältnis zwischen Therapeut und Patient beschäftigten spätere Generationen und sind immer noch nicht ganz entschieden. 


\section{Anmerkungen}

1 Friedhelm Auhuber, In einem fernen dunklen Spiegel: E. T. A. Hoffmanns Poetisierung der Medizin (Opladen, 1986); Wulf Segebrecht, Krankheit und Gesellschaft: Zu E.T. A. Hoffmanns Rezeption der Bamberger Medizin, in: Romantik in Deutschland, Sonderband der Deutschen Vierteljahresschrift, hg. von Richard Brinkmann (Stuttgart, 1978, pp. 267-90); Georg Reuchlin, Das Problem der Zurechnungsfähigkeit bei E.T. A. Hoffmann und Georg Büchner. Zum Verhältnis von Literatur, Psychiatrie und Justiz im frühen neunzehnten Jahrhundert (Frankfurt, 1985). S. auch die Anmerkungen zu E.T.A.Hoffmann, Die Serapions-Brüder, ed. von Hans-Joachim Kruse (Berlin/Weimar, 1978), Bd.1, 2.

2 Robert Mühlher, Deutsche Dichter der Klassik und Romantik (Wien, 1976), S. 275-6; Herbert M. Mühlpfordt, Vererbungs- und Umwelteinflüsse auf die Brüder J. L. und E.T. Hoffmann, in: Jahrbuch der Albertus-Universität zu Königsberg, (Würzburg) 17 (1967), S. 80-146, S.101; Christa Karoli, E.T.A.Hoffmann und Zacharias Werner, in: Mitteilungen der E. T. A. Hoffmann-Gesellschaft 16 (1970), S.43-61, S. 55.

3 Karoli, Hoffmann, S. 46.

4 S. Klaus Dörner, Bürger und Irre: Zur Sozialgeschichte und Wissenschaftssoziologie der Psychiatrie (Frankfurt, 1969), S.254-55. S. auch Friedrich Roth, D. Adalbert Friedrich Marcus, der erste dirigierende Arzt des allgemeinen Krankenhauses zu Bamberg, in: Festschrift zum 100jährigen Jubiläum des allgemeinen Krankenhauses zu Bamberg (Bamberg, 1889), S. 21-34; Nelly Tsouyopoulos, Die neue Auffassung der klinischen Medizin als Wissenschaft unter dem Einfluss der Philosophie im frühen 19. Jahrhundert, in: Berichte zur Wissenschaftsgeschichte 1 (1978), pp. 87-100, pp.89-94, die eine neue Einstellung der Krankheit gegenüber bemerkt, als Resultat des Einflusses von Brown und anderen, nämlich Einheit und nicht Antithese zwischen dem gesunden und pathologischen $\mathrm{Zu}$ stand; Friedrich Speyer, Dr. A. F. Marcus nach seinem Leben und Wirken (Bamberg, 1817); Karl E. Rothschuh, Naturphilosophische Konzepte der Medizin aus der Zeit der deutschen Romantik, in: Romantik in Deutschland, S.243-66, bes. S.248. Segebrecht, Krankheit, bietet einen ausführlichen Hintergrund über die medizinischen Fachkreise in Bamberg während Hoffmanns dortigen Aufenthalts sowie Hoffmanns Leseprogramm auf dem Gebiet der Medizin. S. auch Eckart Klessmann, E. T. A. Hoffmann oder die Tiefe zwischen Stern und Erde: Eine Biographie (Stuttgart, 1988), S. 223-5.

5 Franz G.Alexander, Sheldon T. Selesnick, The History of Psychiatry: An Evaluation of Psychiatric Thought and Practice from Prehistoric Times to the Present (New York, 1966), S. 123; Werner Leibbrand, Romantische Medizin (Leipzig, 1939), S.145-46. S. auch Gesa Wunderlich, Krankheits- und Therapiekonzepte am Anfang der deutschen Psychiatrie (Diss., Berlin, 1981), S. 7-14.

6 S.Dörner, Bürger und Irre, S. 255.

7 Dörner, Bürger, S. 256.

8 Karl E. Rothschuh, Naturphilosophische Konzepte der Medizin aus der Zeit der deutschen Romantik, in: Romantik in Deutschland, S.243-66, S. 244.

9 Schubert, Ansichten, S. 302, 363. Zu diesem Thema schreibt Schubert: «Es werden zwar die noch ungeborenen Kräfte eines künftigen Daseyns vornehmlich in einem krankhaften oder ohnmächtigen Zustand des jetzigen sichtbar [...]. [...] mag auch im gesunden und wachen Zustand der vollkommeneren Organe jene dunklere Sympathie entfernter Wesen immer wirksamer seyn, sie vermag aber erst in solchen Momenten wie die des magnetischen 
Schlafs, des Nachtwandelns, Wahnsinns und andern ähnlichen krankhaften Zuständen hervorzutreten.» S. auch S. 204. Das Porträt Serapions hat viele Ähnlichkeiten mit Schuberts Auffassung des «versteckten Poeten». S. auch Armand DeLoecker, Zwischen Atlantis und Frankfurt (Frankfurt, 1983), S.24.

10 Dörner, Bürger und Irre, S. 256.

11 S.Sigmund Freud, Collected Papers, 1917, übers. von Joan Riviere, Bd.IV, S. 155-56.

12 Karl Ochsner, E. T. A. Hoffmann als Dichter des Unbewussten: Ein Beitrag zur Geistesgeschichte der Romantik (Frauenfeld, 1936), S.51.

13 G. von Maasen, E.T.A.Hoffmanns Sämtliche Werke (München/Leipzig, 1912), Bd.VI, S. 10-11.

14 Carl Alexander Friedrich Kluge, Versuch einer Darstellung des animalischen Magnetismus als Heilmittel (Berlin, 1811), hebt den Einfluss des Magnetiseurs auf den Patienten immer wieder hervor, wie z.B. in den folgenden Zitaten: «Vorzüglich wohl fühlen sie sich in der Nähe ihres Magnetiseurs, der gleichsam ein zweites Ich ihres magnetischen Lebens ist.» (S.207); «tritt er jetzt [...] auch unter die Herrschaft des Willens seines Magnetiseurs» (S.228). Auf S. 230 behauptet Kluge, dass der Magnetisierte dem Willen des Magnetiseurs nicht widerstehen könne, selbst wenn er wach sei. Da die damaligen Ärzte so stark daran glaubten, überrascht es nicht, dass die negativen Möglichkeiten dieser Behandlung Hoffmann Angst einjagten.

15 Schubert, Ansichten, S. 334-7, 340. Franz Anton Mesmer, Mesmerismus : oder System der Wechselwirkungen, Theorie und Anwendung des thierischen Magnetismus als die allgemeine Heilkunde zur Erhaltung des Menschen (Berlin, 1814), red. von Karl Christian Wolfart. (Neudruck Amsterdam, 1966), S. 199-209.

16 H. B. Gibson, Hypnosis: Its Nature and Therapeutic Uses (London, 1977), S. 38.

17 Z.B.: Mesmer, Mesmerismus, S. 199-200; Schubert, Ansichten, S. 340-60.

18 Alle Hoffmann-Zitate stammen aus der folgenden Ausgabe: E.T. A. Hoffmann, Die Serapionsbrüder (München) hrsg. von W. Müller-Seidel; Texthinweise erscheinen in Klammern. Der Glaube, dass der Kranke in einem exaltierten geistigen Zustand war, erscheint oft zu der Zeit in Fachschriften. Zu diesem Thema s. Mesmer, Mesmerismus, S. 199-200; Schubert, Ansichten, S.340-360; Leibbrand, Romantische Medizin, S.136; Peter Schmidt, Gesundheit und Krankheit in romantischer Medizin und Erzählkunst, in: Jahrbuch des freien deutschen Hochstifts 1966, S.197-228, S.210-211. S. auch Segebrecht, Krankheit, S. 276 .

19 Man könnte dabei die verschiedenen Schicksale Maries (Nussknacker und Mausekönig) und Wolframs von Eschenbach (Kampf der Sänger) mit dem von Fröbom (Die Bergwerke zu Falun) in diesem Werk vergleichen.

20 Olga Randl hat früh in ihrer Dissertation, Das Dämonische als Stilform in den literarischen Werken E.T.A. Hoffmanns (München, 1912), die Verbindung zwischen dem magnetischen Zustand und Wahnsinn bemerkt (S.21). S. auch Natalie Reber, Studien zum Motiv des Doppelgängers bei E. T. A. Hoffmann (Giessen, 1964), S. 209.

21 Leibbrand, Romantische Medizin, S. 134: «Ferner schien die eigene Individualität in die des Magnetiseurs einzugehen.» Schubert, Ansichten, S. 344-5: «Es bleibt nun vorzüglich nur noch eine Erscheinung des magnetischen Schlafs übrig, die ohnstreitig zu den merkwürdigsten unter allen gehört, jene tiefe Sympathie der Somnambule mit dem Magnetiseur und andern mit ihr und ihm im Rapport stehenden Personen.» S. 344. 
Heinz Otto Bürger, Arzt und Kranker in der deutschen schönen Literatur des 19. Jahrhunderts, in: Der Arzt und der Kranke in der Gesellschaft des 19. Jahrhunderts (Stuttgart, 1967), S. 98-106, verbindet die Darstellung des Magnetiseurs als eines bösen Arztes mit der negativen literarischen Tradition, was die Darstellung des Mediziners betrifft. S. auch Peter Schmidt, Gesundheit und Krankheit in romantischer Medizin und Erzählkunst, in : Jahrbuch des freien deutschen Hochstifts 1966, S. 197-228, und Mühlher, Deutsche Dichter, S. 361 .

22 S. S.693, Werke. Herman Meyer, Der Sonderling in der deutschen Dichtung (München, 1963), S. 112 und Edgar Marsch, Die Kriminalerzählung: Theorie, Geschichte, Analyse (München, 1972), S.148, besprechen die Problematik eines einseitigen künstlerischen Wahnsinns.

23 Klaus D. Post, Kriminalgeschichte als Heilsgeschichte, in : Zeitschrift für deutsche Philologie 95 (1976), S. 132-56, S. 133, schreibt, dass Cardillac, wie Torbern, den tellurischen Mächten anheimgefallen sei. Helmut Himmel, Schuld und Sühne der Scuderi, in: Mitteilungen der E.T.A.Hoffmann-Gesellschaft, Bd.7 (1960), S.1-15, S.7, 9, 10, sieht die Ähnlichkeit zwischen den zwei Erzählungen, zieht jedoch weder den Magnetismus noch die Metalle in Betracht. S. auch Peter von Matt, Die Augen der Automaten. E. T. A. Hoffmanns Imaginationslehre als Prinzip seiner Erzählkunst (Tübingen, 1971), S.127 F. Kenneth Negus, E.T. A. Hoffmanns Other World: The Romantic Author and his «New Mythology» (Philadelphia, 1965), vergleicht die zwei Geschichten und bemerkt, dass die symbolischen Materialien beider Unterwelten ähnlich sind, erwähnt aber den Magnetismus nicht.

24. Schubert, Ansichten, 13. Kapitel, besonders S.336-7 und 355-6. Himmel, Schuld, sieht einen Zusammenhang, geht ihm aber nicht nach.

25 Kluge, Versuch, S.166. S. auch S. 162. 164, 447.

26 Kritische Arbeiten über vorgeburtliche Einflüsse und die Rolle des Ritters schliessen folgende Werke ein: Otto Nipperdey, Wahnsinnsfiguren bei E.T. A. Hoffmann (Diss., Köln, 1957) S.108; J. M.Ellis, E.T.A.Hoffmanns ‘Fräulein Scuderi〉, Modern Language Notes, Bd. 64 (1969), S. 340-50; Hermann F. Weiss, The Labyrinth of Crime: A Reinterpretation of E.T.A. Hoffmann's «Das Fräulein von Scuderi), in: Germanic Review, 51 (1976, S. 18089, besonders S. 182-3; Post, Kriminalgeschichte, S. 148.

27 In seiner Besprechung von Kluge deutet J. McGlathery, Mysticism and Sexuality: E.T.A. Hoffmann, Part I: Hoffmann and his Sources (Las Vegas, 1981), S. 158 an, dass Magnetismus hier zwischen dem Ritter und Cardillacs Mutter ins Spiel käme, verbindet ihn aber nicht mit Schubert und geht dem Problem nicht nach. S. auch Marianne Thalmann, Der Trivialroman des 18. Jahrhunderts (Liechtenstein, 1967), S. 203.

28 S. z. B. den Leichnam des toten Grafen in: Der unheimliche Gast.

$29 \mathrm{Zu}$ dieser Frage gab es Kontrahenten auf beiden Seiten. Dagegen schrieb Christian Rickmanns Von der Unwahrheit des Versehens (Jena, 1770). Genauso passioniert dafür war D. H. G. Wüstneis Verteidigung der Theorie, die 1809 in Rostock erschien: Versuch über die Einbildungskraft der Schwangeren in Bezug auf ihre Leibesfrüchte zur Beantwortung der Frage: «Können Schwangere sich wirklich versehen?» Wüstnei glaubt, dass Seele und Körper in einer derart intimen Beziehung stünden, dass solche Einflüsse zustande kommen müssten. Zwar weiss man nicht, ob Hoffmann mit dieser Arbeit vertraut war, aber die Darstellung der Theorie entspricht der weitverbreiteten Meinung der damaligen Zeit. Kluge, Versuch, glaubte auch daran: S. S. 352-4, 473. Gisela Gorski, E.T.A.Hoffmann: 
Fräulein von Scuderi (Stuttgart, 1980), S. 151, dass der magnetische Blick auf das Gold und die Juwelen übertragen wird.

30 Hellmuth Himmel, Schuld und Sühne der Scuderi: Zu Hoffmanns Novelle, S. 215-236, S. 227 in: E.T.A. Hoffmann (Darmstadt, 1976); Lothar Pikulik, E.T.A.Hoffmann als Erzähler (Göttingen, 1987), in einer sonst anregenden Analyse, p. 174; für eine kommentierte Bibliographie der umfangreichen Literatur über Cardillac, s. Brigitte Feldges und Ulrich Stadler, E.T. A. Hoffmann: Epoche-Werk-Wirkung (München, 1986), S. 154-157. 


\section{Summary}

The mentally disturbed artist in the work of E.T.A.Hoffmann against the background of psychological theory of the time

The work of one of the greatest writers of German Romanticism, E. T. A. Hoffmann, incorporates a great deal of current medical knowledge, which Hoffmann used in a skilful and detailed manner in the portrayal of his characters and their motives. Immersed as he was in contemporary medical practice, an interest fuelled by his own deep-seated hypochondria, he was particularly taken with the works of Philippe Pinel, Johann Christoph Reil, Carl A.F. Kluge, and Gotthelf Heinrich Schubert, who were all interested in the working of the mind. This article demonstrates how attention to Hoffmans's medical reading list offers insights useful for critical understanding of his work, using as an example the analysis of the mad goldsmith Cardillac in one of Hoffmann's most famous stories, «Das Fräulein von Scuderi〉.

\section{Résumé}

\section{L'artiste aliéné dans l'œuvre d'E.T. A. Hoffmann vu dans le contexte de la psychologie de l'époque}

L'œuvre d'E.T. A. Hoffmann, l'un des plus grands écrivains du romantisme allemand, contient une grande quantité d'informations médicales courantes, que Hoffmann utilisait de manière habile et détaillée dans le portrait de ses personnages et de leurs intentions. Plongé comme il l'était dans la pratique médicale contemporaine, un intérêt nourri par sa propre hypocondrie profonde, Hoffmann était particulièrement impressionné par les travaux de Philippe Pinel, Johann Christoph Reil, Carl A.F. Kluge et Gotthilf Heinrich Schubert, qui tous s'intéressaient au fonctionnement de l'esprit. Cet article, qui prend comme exemple l'analyse de l'orfèvre fou Cardillac dans l'une des histoires les plus connues de Hoffmann, «Das Fräulein von Scuderi», démontre comment l'étude de la liste de lectures médicales de Hoffmann peut aider à la compréhension critique de son œuvre.

Professor Vickie Ziegler, Ph. D

The Pennsylvania State University, Dept. of German

S-323 Burrowes Building South

University Park

Pennsylvania 16802

USA 


\section{Bicentenaire de Lavoisier}

\section{Première annonce}

Pour commémorer la mort de Lavoisier survenue le 8 mai 1794, le Comité Lavoisier de l'Académie des Sciences, organise à cette occasion, du 3 au 6 mai 1994 diverses manifestations.

Elles comprendront, à Paris, un Colloque Européen qui se tiendra à la Maison de la Chimie, puis une visite au Laboratoire de Lavoisier présenté au musée des Techniques.

La journée du 6 mai se déroulera dans la région de Blois où Lavoisier possédait une grande propriété et sera consacré à ses activités agronomiques et politiques.

Pour tout renseignement et suggestions éventuelles s'adresser à

Monsieur Horeau Alain

Madame Michèle Goupil

Président du Comité du ou à Secrétaire du Comité

Bicentenaire Lavoisier

Académie des Sciences, 23, Quai Conti, F-75006 Paris 